\title{
Surface segregation in Cu-Ni alloys
}

\author{
National Aeronautics Good \\ National Aeronautics and Space Administration, Lewis Research Center, Cleveland, Ohio 44135 \\ Guillermo Bozzolo
National Aeranautics and Space Administration, Lewis Research Center, Cleveland, Ohio 44135
and Analex Corporation, 3001 Aerospace Parkway, Brook Park, Ohio $44142-1003$ \\ and Analex Corporation, 3001 Aerospace Parkway, Brook Park, Ohio 44142-1003 \\ $\because A-26-7.26$

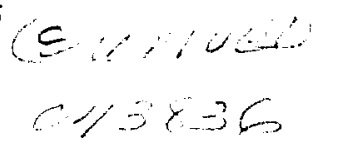

$1+5$

John Ferrante

National Aeronautics and Space Administration, Lewis Research Center, Cleveland, Ohio 44135

(Received 23 June 1993)

\begin{abstract}
Monte Carlo simulation is used to calculate the composition profiles of surface segregation of $\mathrm{Cu}-\mathrm{Ni}$ alloys. The method of Bozzolo, Ferrante, and Smith [Phys. Rev. B 45, $493(1992)$ ] is used to compute the
energetics of these systems as a function of temperature, crystal face, and bulk concentration. dictions are compared with other theoretical and experimental results.
\end{abstract}

Early experimental evidence obtained from work. function measurements showed that the surface composition of evaporated $\mathrm{Ni}-\mathrm{Cu}$ alloy films was constant, and that the surface was enriched in $\mathrm{Cu}$ over a wide range of bulk compositions. ${ }^{1-3}$ These results were confirmed with subsequent experimental evidence obtained in a broader range of temperatures, ${ }^{4}$ and supported by hydrogen adsorption and benzene hydrogenation measurements on $\mathrm{Cu}-\mathrm{Ni}$ films. ${ }^{3,5,6}$ The behavior of alloy powders sintered at $T=600 \mathrm{~K}$ indicated that conclusions made for films were valid for powders as well. ${ }^{7-9}$ Soon thereafter, a controversy between Auger-electron spectroscopy (AES) data in the region $0.8-1.0 \mathrm{keV}$, and volumetric adsorption measurements with $\mathrm{CO}$ at room temperature, ${ }^{10}$ was resolved by the AES experiments of Helms and coworkers. " These experiments, while contradicting the analysis of Takasu and Shimizu ${ }^{12}$ that established surface enrichment of the element with the minority composition in the bulk, clearly determined the $\mathrm{Cu}$ enrichment of the surface for a wide range of bulk compositions. These results were later confirmed by the AES work of Kuijers and Ponec, ${ }^{13}$ and the analysis of surface segregation and in-depth surface profiles done from a calculation on the basis of Palmberg's physical mechanism. ${ }^{14}$

The composition depth profile was at this point uncertain: although most results favored $\mathrm{Cu}$ enrichment of planes below the surface, ${ }^{15,16}$ others indicated a monotonic decrease in $\mathrm{Cu}$ composition ${ }^{17}$ or oscillations. ${ }^{18}$ More precise results based on time-of-flight atoms-probe (AP. FIM) experiments by $\mathrm{Ng}$ and co-workers ${ }^{19}$ indicated surface composition dependence on the crystal face, as well as evidence of a monotonic decrease in $\mathrm{Cu}$ concentration for planes below the surface, returning to bulk composition after five layers. Other experimental techniques used include low-energy ion scattering (LEIS), ${ }^{20}$ particularly sensitive to the outermost atomic layer of the surface. and $x$-ray photoelectron spectroscopy (XPS). ${ }^{21}$ The XPS analysis of Webber et al. ${ }^{10}$ provided important evidence of $\mathrm{Cu}$ segregation in Ni-rich alloys. The XPS experiments of $W$ andelt and Brundle ${ }^{21}$ also provided evidence
for crystal face specificity.

During this same period, there was extensive theoretical work devoted to the problem of surface segregation in alloys, including early calculation models for binary alloys, ${ }^{22}$ the regular solution model, ${ }^{23}$ calculations based on bulk strain energy considerations, ${ }^{22,24}$ and the role of thermodynamic parameters of alloys. ${ }^{25,26}$ Microscopic electronic theories were also applied to explain $\mathrm{Cu}$ enrichment, ${ }^{27}$ as were semiempirical methods [the embeddedatom method (EAM) (Ref. 28)] and Monte Carlo techniques, ${ }^{29,30}$ which also provided information on clustersize distribution at the surface.

More recently, Sakurai et al. ${ }^{31}$ reported AP-FIM results on the surface composition of $\mathrm{Cu}-\mathrm{Ni}$ alloys which indicated $\mathrm{Cu}$ depletion on the surface for alloys containing more than 84 at. \% for $\mathrm{Cu}$-rich alloys, ${ }^{32}$ showing that no such reversal of the segregation species occurs, in accordance with the body of previously available experimental and theoretical evidence, ${ }^{1-30}$ together with two recent theoretical studies confirming those results. ${ }^{33,34}$

We report the application of a semiempirical method for alloys recently developed by Bozzolo, Ferrante, and Smith (BFS), ${ }^{35}$ which builds on ideas underlying the equivalent crystal theory (ECT). ${ }^{36}$ This method has been applied to a variety of fundamental problems in alloy structure providing, in all cases, excellent agreement with experiment. ${ }^{35}$ Here we focus on the study of surface composition and in-depth profiles of $\mathrm{Cu}-\mathrm{Ni}$ alloys, for different crystal faces, by means of a Monte Carlo calculation in which the alloy energetics are described by BFS.

The BFS method is based on the idea that the energy of formation of an arbitrary alloy structure is the superposition of individual contributions $e_{i}$ of nonequivalent atoms
in the alloy:

$$
e_{i}=e_{i}^{S}+g_{i}\left(e_{i}^{C}-e_{i}^{C_{0}}\right) \text {. }
$$


for the actual geometrical distribution of the atoms surrounding atom $i$, computed as if all its neighbors were of the same atomic species, and a chemical energy $e^{C}$, which takes into account the fact that some of the neighbors of atom $i$ may be of a different chemical species. The coupling function $g_{i}$, ensures the correct asymptotic behavior of the chemical energy contribution. The strain energy of a pure defect crystal is calculated assuming that every neighbor of atom $i$ is of the same species $X . e_{i}^{S}$ is then computed with any technique (first-principles methods, semiempirical techniques, etc.). For $e_{i}^{C}$ we interpret the chemical composition as a defect of an otherwise pure crystal. We represent this defect by "perturbing" the electronic density of the overlap region between dissimilar atoms, and locating them at equilibrium lattice sites of atom $i$. The ideas of equivalent crystal theory (ECT) are used to develop a procedure for the evaluation of the energy associated with this "defect." To free the chemical energy of the structural defect energy, which should be included only in the strain energy, we reference $e_{i}^{C}$ to a similar contribution where no such perturbation is included $\left(e_{i}^{C_{0}}\right)$. Finally, the coupling function $g_{i}$ is defined as $g_{i}=e^{-a_{i}^{S}}$, where $a_{i}^{S}$ is a solution of $e_{i}^{S}=E_{C}^{i}\left[1-\left(1+a_{i}^{S}\right) \exp \left(-a_{i}^{S}\right)\right]$, and where $E_{C}^{i}$ is the cohesive energy for atom $i$. We direct the reader to Ref. 35 , where a detailed description of the calculation of strain and chemical energy contributions is provided. Except for two parameters determined by fitting to experimental or theoretical alloy properties, the method relies on pure element properties. Moreover, the pairwise character of the interaction between dissimilar species facilitates application to multicomponent systems with no further experimental or theoretical input. Within the framework of BFS, the calculation of defect energies requires information on the atomic positions only. The input parameters are readily available for a variety of fcc and bcc alloys. The experimental input used for the $\mathrm{Cu}-\mathrm{Ni}$ alloy is the heat of solution in the dilute limit, $E^{\mathrm{sol}}[\mathrm{Cu}(\mathrm{Ni})]=0.03$ and $E^{\mathrm{sol}}[\mathrm{Ni}(\mathrm{Cu})]=0.09 \mathrm{eV}$. The BFS parameters thus obtained, together with other binary systems, are detailed in Ref. 35 .

The procedure used to determine the segregation profiles is a variant of the Metropolis Monte Carlo method. Initially a computational cell consisting of 15 layers of 98 atoms [(100) and (111) surfaces], or 30 layers of 49 atoms [(110) surface] is set up. A starting composition is chosen, and each atom within the cell is assigned a species probabilistically, so that the initial composition is uniform, both throughout the cell and within each layer individually. All but the last four layers are "active" in that their compositions are allowed to vary during the computation. For reasons having to do with the details of the energy calculations, the last four layers are static, and change neither composition nor the detailed distribution of the two chemical species within each layer.

As the simulation proceeds, a pair of atoms of opposite species is chosen randomly from within the active region of the computational cell, and the total energy of the cell is computed. The chemical species of the two atoms are reversed, and the total energy is recomputed. The rever- sal is accepted according to the Metropolis criterion (it is accepted if it lowers the energy, or with probability $e^{-\Delta E / k T}$ if it raises the energy by an amount $\Delta E$ ). The simulation is continued until the segregation profile attains a steady state.

Note that the procedure as described results in a depletion of the segregating species in all but the top few layers. A variety of replenishment algorithms have been used elsewhere; in this work, however, we use a computational cell containing enough layers that the asymptotic concentration far from the surface is evident, and take the bulk concentration to be that value. Atomic relaxation, which we have ignored, can affect the results in some cases. Previous work by the authors ${ }^{35}$ on singleimpurity-atom segregation has shown that when there is a large lattice constant mismatch between the species (Cu-Ni has a small lattice mismatch), the segregation energy is substantially different when computed with and without relaxation; the strain component of the energy is dominant. Still, it is expected that near the surface, where the concentration differs appreciably from the bulk, relaxation may reduce the strain component of the energy, and result in a modification of the segregation profile. This issue is being investigated further.

Our goal is to present a set of results addressing the different issues covered in previous studies, concerning the dependence of the profiles on bulk concentration, temperature, and crystal face, as well as specific features of the profiles. In agreement with experiment, ${ }^{1-21}$ we found that there is no segregation reversal for high $\mathrm{Cu}$ concentrations as found by Sakurai et al. ${ }^{31}$ or depletion in the second layer as obtained with EAM. Moreover, the surface concentrations are in excellent agreement

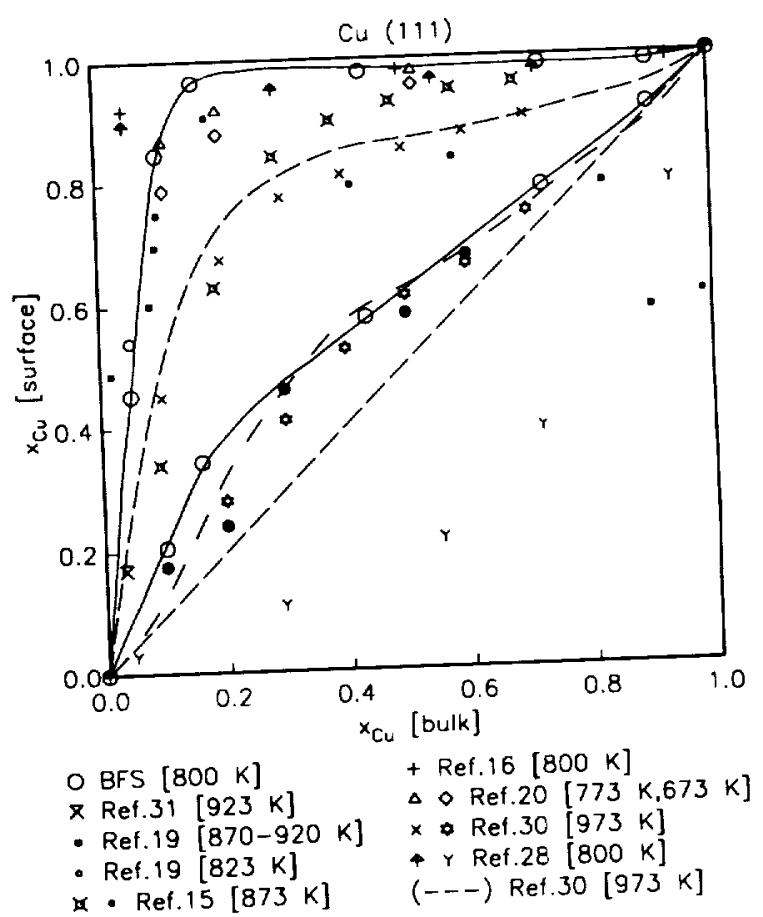

FIG 1. $\mathrm{Cu}(111)$ surface composition vs $\mathrm{Cu}$ bulk composition: comparison between the experimental and theoretical results for the first and second planes. 
with experimental values, in particular for the (111) face, the subject of most experimental studies. Figure 1 displays our predictions for the (111) surface concentration, as well as several experimental measurements and other theoretical approaches. Our results for the composition of the surface plane and the first plane below the surface were obtained at the fixed temperature $T=800$ $K$. BFS predicts the formation of a solid solution for the bulk alloy for temperatures between 300 and $400 \mathrm{~K}$. Following Foiles, ${ }^{28}$ we also computed the Warren-Cowley short-range order parameters ${ }^{37}$ as a function of concentration for a wide range of temperatures. These results are comparable to experimental values, and clearly indicate the formation of the solid solution for temperatures above $400 \mathrm{~K}$. Therefore, the temperature chosen for this calculation is well within the continuous solid solution region of the phase diagram predicted by our model (similar to that used in other theoretical calculations, and within the range of experimental measurements). Our results seem to be particularly good for low bulk concentrations, where there is a rapid increase in the surface concentration.

Figure 2 shows the segregation profiles as a function of the bulk concentration and crystal face. We find a direct relationship between the surface concentration and the roughness of the surface (defined as the reciprocal of the surface packing fraction for hard spheres). This proportionality is also seen in the "thickness" of the surface region, i.e., the planes near the surface where the concentration differs considerably from the bulk value. Our results are in quantitative agreement with experiment ${ }^{21}$ and other theoretical calculations. ${ }^{28-30}$ Our results (Fig. 3) for the temperature dependence of the profiles agree qualitatively with a previous Monte Carlo study by Donnelly and King. ${ }^{29}$ The BFS values (Fig. 2) disagree quantitatively with the results of Ref. 29 , in part due to the fact that their surface layer is defined as consisting of atoms which are not fully coordinated. Thus, using the same definition for the first atomic layer in both cases, the actual $\mathrm{Ni}$ composition on the first atomic layer is in good agreement with our predictions. Finally, Fig. 4 shows the

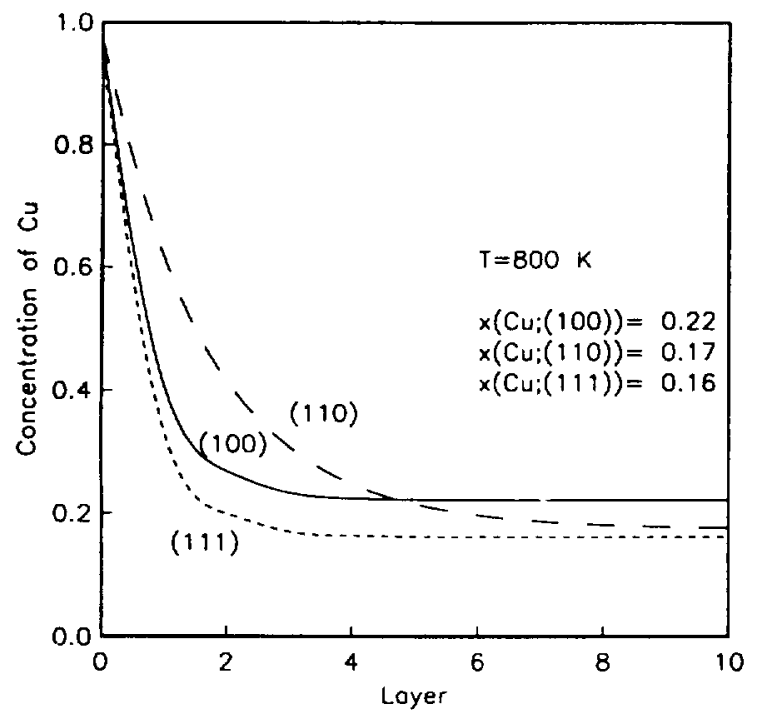

FIG. 2. Cu composition depth profiles for the (111), (110), and $(100)$ surface of $\mathrm{Cu}-\mathrm{Ni}$ alloys.

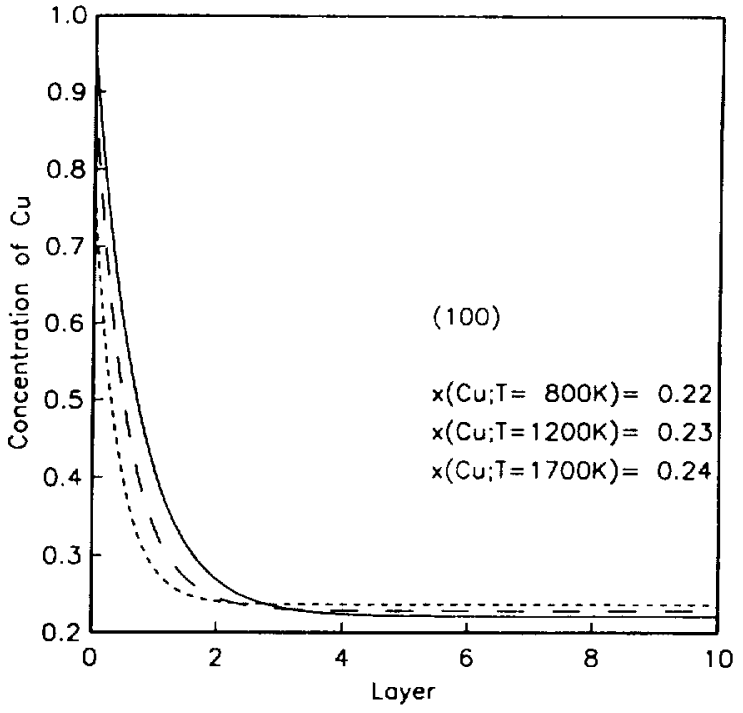

FIG. 3. Cu composition depth profiles as a function of temperature.
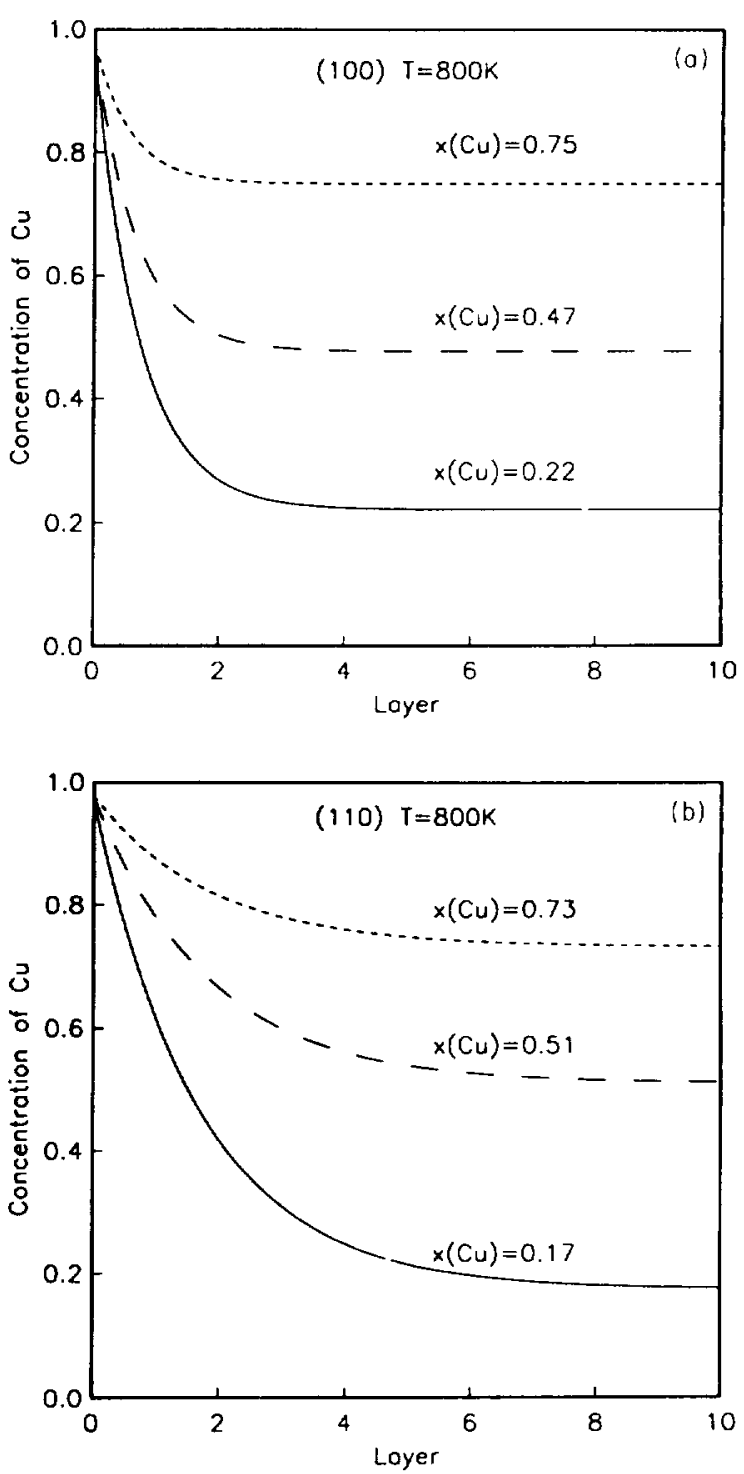

FIG. 4. Cu composition depth profiles for different bulk concentrations for the (a) (100) and (b) (110) $\mathrm{Cu}-\mathrm{Ni}$ surfaces. 
segregation profiles for two different orientations and several bulk concentration values. Consistent with previous theoretical calculations, the composition approaches the bulk alloy within a few atomic layers, regardless of the bulk concentration value. On the other hand, the surface concentration values are consistent with those found experimentally for the (111) face (see Fig. 1).

In this paper we examined several points which we believe are of interest to the community at large: we demonstrated the ability of our semiempirical method to describe this type of phenomenon. We also provided a comprehensive study of the segregation profiles in terms of temperature, crystal face, and bulk concentration. The temperatures and bulk concentration values used correspond to the solid solution region of the $\mathrm{Cu}-\mathrm{Ni}$ phase diagram. Our results are in agreement with several previous studies that indicate a monotonic profile for all temperatures and concentrations, with no evidence of segregation reversal.

\section{ACKNOWLEDGMENTS}

Fruitful discussions with Dr. N. Bozzolo are gratefully acknowledged. This work was partially supported by the Engineering Directorate, NASA Lewis Research Center.
'W. M. H. Sachtler and G. J. H. Dorgelo, J. Catal. 4, 654 (1965). ${ }^{2}$ W. M. H. Sachtler and R. Jongepier, J. Catal. 4, 665 (1965).

${ }^{3}$ P. van der Plank and W. M. H. Sachtler, J. Catal. 12, 35 (1968). ${ }^{4}$ P. E. C. Franken and V. Ponec, J. Catal. 42, 398 (1976).

${ }^{5}$ W. M. H. Sachtler and P. van der Plank, Surf. Sci. 18, 62 (1969).

${ }^{6}$ V. Ponec and W. M. H. Sachtler, J. Catal. 24, 250 (1972).

7J. M. Beelen, V. Ponec, and W. M. H. Sachtler, J. Catal. 28, 376 (1973); A. Roberti, V. Ponec, and W. M. H. Sachtler, ibid. 28, 381 (1973).

${ }^{8}$ D. A. Cadenheah and N. J. Wagner, J. Phys. Chem. 72, 2775 (1968); J. H. Sinfelt, J. L. Carter, and D. J. C. Yates, J. Catal. 24, 283 (1974).

${ }^{9}$ M. Araki and V. Ponec, J. Catal. 44, 439 (1976); W. A. A. van Barneveld and V. Ponec, Rec. Trav. Chim. 93, 243 (1974).

${ }^{10} \mathrm{G}$. Ertl and J. Küppers, J. Vac. Sci. Technol. 9, 829 (1972); Surf. Sci. 24, 104 (1971); D. T. Quinto, V. S. Sundaram, and W. D. Robertson, ibid. 28, 504 (1971); G. D. Lyubarskii, Problemy Kinetika i Kataliz 14, 129 (1970); S. Engels, G. Höfer, J. Höfer, J. Radke, and M. Wilde, Z. Chem. 15, 459 (1975).

${ }^{11}$ C. R. Helms, K. Y. Yu, and W. E. Spicer, Surf. Sci. 52, 217 (1975); C. R. Helms, J. Catal. 36, 114 (1975); C. R. Helms and K. Y. Yu, J. Vac. Sci. Technol. 12, 276 (1975).

${ }^{12}$ Y. Takasu and H. Shimizu, J. Catal. 29, 479 (1973).

${ }^{13}$ F. J. Kuijers and V. Ponec, Surf. Sci. 68, 294 (1977).

${ }^{14}$ P. W. Palmberg, Anal. Chem. 45, 549A (1973).

${ }^{15} \mathrm{~K}$. Watanabe, M. Hashiba, and T. Yamashina, Surf. Sci. 61, 483 (1976).

${ }^{16}$ P. R. Webber, C. E. Rojas, P. J. Dobson, and D. Chadwick, Surf. Sci. 105, 20 (1981).

${ }^{17}$ P. J. Durhan, R. G. Jordan, G. S. Sohal, and L. T. Wille, Phys. Rev. Lett. 53, 2038 (1984).

${ }^{18}$ D. T. Ling, J. N. Miller, I. Lindau, W. E. Spicer, and P. M. Stefan, Surf. Sci. 74, 612 (1978).

${ }^{19}$ Y. S. Ng, T. T. Tsong, and S. B. McLane, Jr., Phys. Rev. Lett. 42, 588 (1979); Surf. Sci. 84, 31 (1979); Y. S. Ng, S. B. McLane, Jr., and T. T. Tsong, J. Vac. Sci. Technol. 17, 154 (1980).

${ }^{20}$ H. H. Brongersma and T. M. Buck, Surf. Sci. 53, 649 (1975);
H. H. Brongersma, M. J. Sparnay and T. M. Buck, ibid. 71, 657 (1978).

${ }^{21} \mathrm{~K}$. Wandelt and C. R. Brundle, Phys. Rev. Lett. 46, 1529 (1981).

${ }^{22}$ G. A. Somorjai and S. H. Overbury, Faraday Disc. Chem. Soc. 60, 279 (1975); P. Wynblatt and R. C. Ku, Surf. Sci. 65, 511 (1977); R. A. van Santen and M. A. M. Boersma, J. Catal. 34, 13 (1974).

${ }^{23}$ F. L. Williams and D. Nason. Surf. Sci. 45, 377 (1974).

${ }^{24}$ V. S. Sundaram and P. Wynblatt, Surf. Sci. 52, 569 (1975).

${ }^{25}$ J. J. Burton, E. Hyman, and D. G. Fedak, J. Catal. 37, 106 (1975); J. J. Burton and E. Hyman, ibid. 37, 114 (1975).

${ }^{26}$ S. H. Overbury, P. A. Bertrand, and G. A. Somorjai, Chem. Rev. 75, 547 (1975); F. F. Abraham and C. R. Brundle, J. Vac. Sci. Technol. 18, 506 (1981); M. J. Kelly and V. Ponec, Prog. Surf. Sci. 11, 139 (1981); A. R. Miedema, Z. Mettal. 69, 455 (1978); J. C. Hamilton, Phys. Rev. Lett. 42, 989 (1979).

${ }^{27}$ G. Kerker, J. L. Moran-Lopez, and K. H. Bennemann, Phys. Rev. B 15, 638 (1977); S. Mukherjee, J. L. Moran-Lopez, V. Kumar, and K. H. Bennemann, ibid. 25, 730 (1982).

${ }^{28}$ S. M. Foiles, Phys. Rev. B 32, 7685 (1985).

${ }^{29}$ R. G. Donnelly and T. S. King, Surf. Sci. 74, 89 (1978).

30J. Eymery and J. C. Joud, Surf. Sci. 231, 419 (1990).

${ }^{31}$ T. Sakurai, T. Hashizume, A. Jimbo, A. Sakai, and S. Hyodo, Phys. Rev. Lett. 55, 514 (1985).

${ }^{32}$ H. H. Brongersma, P. A. J. Ackermans, and A. D. van Langeveld, Phys. Rev. B 34, 5974 (1986).

${ }^{33}$ H. Y. Wang, R. Najafabadi, and D. J. Srolovitz, Phys. Rev. B 45, 12028 (1992).

${ }^{34}$ H. Dreysse, L. T. Wille, and D. de Fontaine, Phys. Rev. B 47, 62 (1993).

${ }^{35}$ G. Bozzolo, J. Ferrante, and J. R. Smith, Phys. Rev. B 45, 493 (1992); G. Bozzolo and J. Ferrante, Scr. Met. Mater. 26, 1275 (1992); Phys. Rev. B 45, 12191 (1992); Scr. Met. Mater. 26, 907 (1992); Phys. Rev. B 46, 8600 (1992); G. Bozzolo, B. Good, and J. Ferrante, Surf. Sci. 289, 169 (1993).

36J. R. Smith and A. Banerjea, Phys. Rev. Lett. 59, 2451 (1987); Phys. Rev. B 37, 10411 (1988); J. R. Smith, T. Perry, A. Banerjea, J. Ferrante, and G. Bozzolo, ibid. 44, 6444 (1991).

${ }^{37}$ J. M. Cowley, Phys. Rev. 77, 669 (1950). 